An explanation of the shape of these curves has been sought, assuming the couple and mantle surrounded by a film of gas through which the heat is conducted. An expression has been obtained that gives the shapes of these curves as obtained experimentally. It has been of assistance in showing the shape of the curves from zero to the smallest diameter.

The theory has also been extended to cover the temperatures assumed by mantles of various emissive powers when placed in a flame of known temperature. Here it has been assumed that the mantle is heated in much the same manner, except that outside the mantle the flame has been cooled in many cases at least nearly to the mantle temperature. This wall of flame protects the mantle from convected and conducted heat losses. The radiation efficiency calculated for the higher temperature mantles agrees with that actually measured experimentally. It has been found that the total emission per unit area per steradian is nearly a linear function of the temperature assumed in a flame. The theory in the light of the experimental curve is interesting as helping to explain the mechanism of a flame heating a mantle placed in it.

\title{
VISUAL DIFFUSIVITY.
}

By Herbert E. Ives.

In developing a theory to account for the behavior of the eye toward intermittent stimuli, the writer was led to postulate for visual mechanism a coefficient of diffusivity, ${ }^{1}$ similar to the coefficient which holds for heat, electrical and all other forms of conduction through matter. By virtue of this finite diffusivity intermittent stimuli are greatly smoothed out before reaching the brain. From the phenomena of flicker it was deduced that this visual diffusivity must vary as the logarithm of the intensity of the stimulus and at a different rate for different colors.

The present study deals with a much simpler case than that of intermittent stimuli, namely that of single flashes of light. The physical illustration is that of instantaneously applied heat sources, a case which has been completely handled mathematically. According to this treatment it follows that if two simultaneously applied stimuli are transmitted by media of different diffusivity

1 "Theory of the Flicker Photometer," Ives and Kingsbury, Philosophical Magazine, November, 1914, p. 708, April, I916, p. 290. 
they will be transmitted at different speeds. In the case of light this would mean that two differently colored simultaneous flashes, for instance red and blue, would be seen as occuring one after the other.

This idea was tested by means of red and blue glasses carried radially on a slowly rotating disc. It was found that the theory was very strikingly borne out, the blue image lagging after the red. The amount of this lag varies with the brightness as it should according to the theory.

On this theory the positive after-image is not a recurrent image, but the record as given by the retinal rods, delayed in transmission by the low diffusivity characteristic of this kind of vision.

\section{A POLARIZATION FLICKER PHOTOMETER.}

\section{By Herbert E. Ives.}

ONE of the most essential features of the flicker photometer is entire absence of mechanical flicker, as caused by the dividing edge between the two fields which are alternated. For this reason some means of eliminating this edge has long been desired.

From another standpoint, that of the theoretical treatment of flickering light, it is highly desirable to have an instrument in which the transition from one light to the other occurs according to some simple mathematical relation. The simplest relation is the sine curve, on which basis the theory of the flicker photometer has been handled by the writer and Mr. Kingsbury. ${ }^{1}$

The polarization flicker photometer recently constructed meets both these demands completely. It consists of a double image prism (Rochon or Wollaston prism), combined with a rotating Nicol prism. The double image prism forms two images of each half of the photometer field, one image being polarized in the horizontal, the other in the vertical plane. The horizontally polarized image of one field is superposed on the vertically polarized image of the other. On observing through the rotating Nicol prism, these two fields dissolve one into the other, following exactly the sine curve relation used in the theoretical work quoted.

Measurements have been made with this new photometer on the critical speeds for disappearance of flicker for various ratios of

1 "The Theory of the Flicker Photometer," Ives and Kingsbury, Philosophical Magazine, November, 1914, p. 708, April, 1916, p. 290. 